\title{
On the Performance of Time-Space Opportunistic Routing in Multihop Mobile Ad Hoc Networks
}

\author{
François Baccelli*, Barłomiej Błaszczyszyn ${ }^{\dagger}$ and Paul Mühlethaler ${ }^{\ddagger}$
}

\begin{abstract}
In classical routing strategies for multihop mobile wireless networks packets are routed on a pre-defined route usually obtained by a shortest path routing protocol. In opportunistic routing schemes, for each packet and each hop, the next relay is found by dynamically selecting the node that captures the packet transmission and which is the nearest to the destination. Such a scheme allows each packet to take advantage of the local pattern of transmissions and fadings at any slot and at any hop. The aim of this paper is to quantify and optimize the potential performance gains of such opportunistic routing strategies compared with classical routing schemes. The analysis is conducted under the following lower layer assumptions: the Medium Access (MAC) layer is a spatial version of Aloha which has been shown to scale well for large multihop networks; the capture of a packet by some receiver is determined by the Signal over Interference and Noise Ratio (SINR) experienced by the receiver. The paper contains a detailed simulation study which shows that such time-space opportunistic schemes very significantly outperform classical routing schemes. It also contains a mathematical study where we show how to optimally tune the MAC parameters so as to minimize the average number of time slots required to carry a typical packet from origin to destination on long paths. We show that this optimization is independent of network density.
\end{abstract}

Index Terms-Network design, stochastic process, simulation, point process, stochastic geometry, optimization, transport capacity, signal to interference ratio, interference, collision, multiple access protocol, MAC layer, geographic routing, cross layer optimization.

\section{INTRODUCTION}

Many studies have been carried out on routing protocols for Mobile Ad Hoc Networks (MANETs), especially at the Internet Engineering Task Force (IETF) in the MANET working group. The aim of these protocols is to build a route maded up of several hops or relay nodes from every source to every destination. Routing protocols are usually subdivided into two classes: reactive protocols and proactive protocols. In reactive protocols the routes are only built on demand. The origin node wishing to obtain a route to a destination floods the network with a request packet. When the destination node receives the request packet, it responds to the origin node and the path used by the request packet determines the route from the origin to the destination node. Ad hoc On-Demand Distance Vector (AODV) and Dynamic Source Routing (DSR) [1], [2] are examples of such protocols. On the other hand, proactive protocols maintain the knowledge of the network topology through the exchange of periodic packets. In such protocols the main issue is to reduce the control overhead.

\footnotetext{
*INRIA/ENS, 45 rue d'Ulm, 75005 Paris FRANCE, Francois.Baccelli@ens.fr

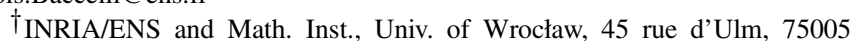
Paris FRANCE, Bartek.Blaszczyszyn@ens.fr

‡INRIA, Le Chesnay, FRANCE, Paul.Muhlethaler@inria.fr
}

When a link state protocol is used, it is important to optimize the broadcast in the network. Optimized Link-State Routing (OLSR) and Topology Dissemination Based on Reverse-Path Forwarding (TBRPF) [3], [4] are examples of such protocols. With all these routing protocols, the route which is selected between a source and a destination is usually a shortest path route, namely the one with the smallest number of hops, and it remains the same for all the packets of the stream.

In the present paper, we analyze yet another class of routing strategies, called opportunistic, where the next relay can be defined at each hop of each packet depending on the local configuration of simultaneous transmitters and the fading or shadowing variables at that time. These strategies imply that we use geographical routing. Instances of such strategies have been considered in e.g. [5], [6] and [7]. The general aim of these opportunistic routing algorithms is to minimize the end-to-end delay required to carry a packet from origin to destination. This metric is more fundamental than simply the number of hops in the route (optimized by the shortest path route), which does not include the time which is wasted in unsuccessful attempts to make a particular hop. The main achievements of the present paper within this context are:

(i) a quantification of the performance gain obtained using opportunistic strategies which are based on geographic routing alone; our simulations show that the average endto-end delay from an origin to some distant destination is at least two and a half shorter for time-space opportunistic routing than for a typical shortest path routing (cf. Observation 4.1).

(ii) a cross-layer optimization of the MAC and routing layers for such strategies; we proved that for long sourcedestination paths, there exists an optimal tuning of that MAC layer that minimizes the average end-to-end delay and that this optimal tuning is the same for all values of node density in the network (cf. Observation 4.3 and Corollary 6.4).

The model used in this paper to address these quantitative questions has the following features:

- it uses a spatial Aloha protocol for the MAC; Aloha [8] along with Time Division Multiple Access (TDMA) was one of the first protocols used in radio networks; one of the main reasons for selecting Aloha here is that it can be tuned in an optimal way that was proved to scale well with node density in [6].

- it uses an SINR criterion for the capture (successful reception) of packets; this model has an information theoretic basis and is used in most papers on the subject, particularly in [9].

The paper is organized as follows. Section II reviews related work and positions the contributions of the present paper in 
this context. Section III gives details on the simulated scenarios and the simulation assumptions. Section IV presents the main observations obtained by the simulations. Some issues related to routing on long paths are discussed in Section V. A mathematical framework for the analysis of the opportunistic routing is developed in Section VI.

\section{State of The ARt, Related Work}

\section{A. Shortest Path Routing and Dijkstra's Algorithm}

Routing protocols are distributed algorithms which select paths along which to send data for all pairs of origin and destination nodes (O-D pairs). Usually, routing protocols send control packets containing topology information to compute the routes. Distance vector and link state routing [10] protocols are the most common within this framework. In link state protocols, each node broadcasts the list of its neighbor nodes within the network. In wireless networks the neighborhood is determined by the transmission range of the radio modem: the neighbors of a node are those at a distance less than this transmission range. Using Dijkstra's algorithm [11], each node running a link state routing protocol can compute the path with the smallest number of hops (called the shortest path below) to any other node. An instance of such a shortest path from the origin node $\mathrm{O}$ to the destination node $\mathrm{D}$ is depicted in Figure 1 (left).

In conventional routing protocols, when a route is established between an origin and a destination, this route remains the same as long as the network nodes do not move and the links are stable.

\section{B. Geographic Routing}

In geographic routing [12], the positions of the nodes are used to determine the route to the destination. Geographic routing is generally proposed more to reduce the routing state of each node than to optimize the routing path from the origin to the destination node. However most of the proposed geographic routing protocols try to optimize geographic criteria. Examples of such criteria can be:

a) Progress towards the destination: in the case of a destination at infinity (say in the $x$ axis direction), one selects a neighbouring receiver that has the largest possible abscissa (see Figure 1 (right)). This was the first approach of geographical routing proposed by Takagi and Kleinrock in 1984 (see [13]); in what follows this approach will be referred to as directional routing.

b) Distance to destination: one selects a neighbouring receiver that is the nearest to the destination (cf [14], [15]; see Figure 1 (left)). This will be referred to as radial routing.

Geographic routing like conventional routing may be used to establish fixed routes between an origin and a destination. In this case the optimal (according to the above geometric criteria) nodes are chosen within a fixed given transmission range of the transmitter (as, for instance, in the shortest path routing). However, the next hop can also be decided when the packet is forwarded. In this case the optimal relays can be selected among the nodes which are in some "dynamic" neighborhood of the transmitters; then the routing is opportunistic in the sense that will be fully explained in the next section.

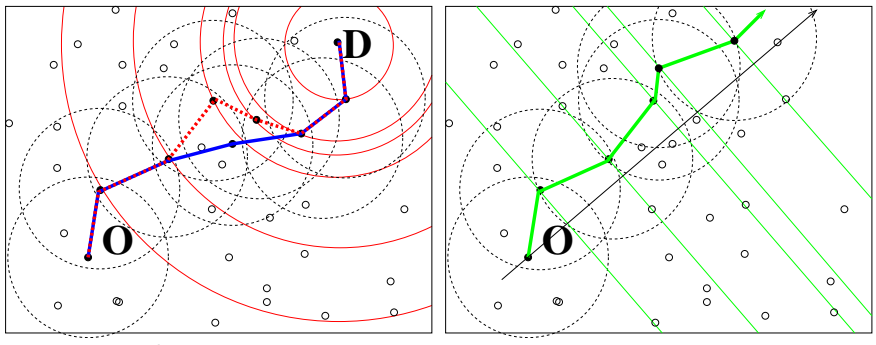

Fig. 1. Left: Shortest path (solid blue curve) versus radial (dashed red curve) routing, with neighborhoods defined by discs of fixed radius (maximum transmission range). Right: Directional routing towards infinity with the same transmission ranges.

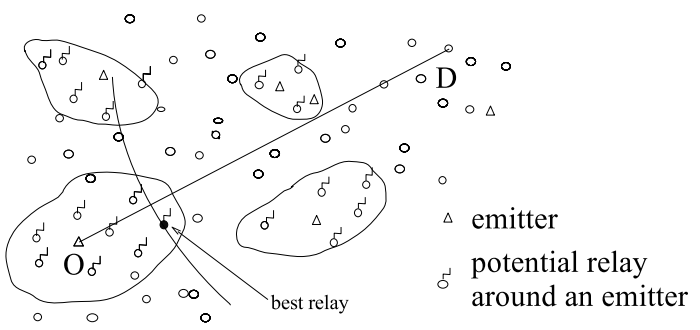

Fig. 2. Firs hop in time-space diversity routing which optimizes the relaying at each time slot.

The following articles propose some form of geographical routing: Greedy Perimeter Stateless Routing (GPSR) in [16]) Implicit Geographic Forwarding (IGF) in [5], and [7], where the distance and the delivery ratio matrix are used to compute the next relay towards the destination.

\section{Time-Space Opportunistic Routing - Mac and Routing Interplay}

Time-space opportunistic routing is based on a dynamic selection of the next relay which takes advantage not only of the local topology of the network but also of the current MAC and channel conditions.

To gain a better understanding of this idea, let us consider a time slot where the MAC layer prescribes a given node to transmit some tagged packet. The set of nodes which transmit in the same slot is random (because of the random primitives in any MAC) as is the fading from any transmitter to any receiver (because of random scatterers and/or motion). Hence the set of receivers which capture this tagged packet according to the SINR condition is also random. Let us define the best relay as the receiver of this random set which captures this tagged packet and which optimizes some geographical criterion. For example, in radial routing, this is the node which is the nearest to the destination of the tagged packet (as depicted in Figure 2, where we represent a part of the network during a time slot with four simultaneous transmissions). This best relay will then be in charge of forwarding the tagged packet further whereas the other receivers of the random set will discard it and refrain from forwarding it any further.

Note that since the set of nodes which transmit in this slot depends on the MAC decisions, which change with time, and on the fading variables, which may also change with time, two tagged packets transmitted from the same given node and with the same destination will in general use different relays.

In order to justify the use of the term "opportunism", let us consider the following scenario where a tagged packet with a given destination $\mathrm{D}$ is currently transmitted by the origin node O under two routing scenarios: 1) the shortest path routing protocol and 2) the time-space opportunistic radial one. With 1 ), the next relay is some fixed node $A$. Under 2), denote by $\mathcal{S}$ 
the random set of receivers which capture the tagged packet. If $A$ belongs to $\mathcal{S}$, then in this time slot 1) will deliver the packet no further from the destination than 2). Indeed $\mathcal{S}$ may contain a node which is closer to the destination than $A$. If $\mathcal{S}$ does not contain $A$, then the same conclusion obviously holds since 1) leaves the packet at $\mathrm{O}$ and $\mathcal{S}$ may contain some node closer to the destination than $\mathrm{O}$. In fact, it may even contain a node closer to the destination than $A$ due to good fading conditions, a situation where opportunism pays off quite substantially. The fact that 2) does better in this time slot than 1) does not of course imply that 2) does better globally on the whole route to the destination. The main aim of the paper is actually to clarify this question and to optimize the global performance of 2).

The implementability of time-space opportunistic routing is discussed in [6] and [5] under the assumption that all nodes have access to a positioning system such as Global Positioning System (GPS). More complete treatment of this problem can be found in [17].

\section{Survey of the Field and Position of our Contributions}

References [5] and [6] seem to be the first where geographic routing has been used in combination with a MAC protocol to provide such a time-space opportunistic routing. Both papers concentrate on directional routing. Reference [7] also uses the idea of opportunistic routing but there are several important differences with respect to the present paper: a) the packets are sent in batches rather than one by one; b) a mixture of geographic and shortest path routings is actually used: directional routing is used far away from the destination and a shortest path mechanism is used for the termination of the routing algorithm close to the destination. Both protocols presented in [5] and [7] use an 802.11 as the MAC access solution. In contrast, the present article uses an Aloha scheme as in [6].

The present paper continues the performance analysis of time-space opportunistic routing that can be found in [6], [12] and [7]. To the best of the authors knowledge, the simulation study of the present paper (Section IV) is the first to address the comparison of a purely geographic routing scheme (radial routing) with shortest path schemes, whereas the simulations of [7] focused on a comparison of the mixed routing scheme proposed there with shortest path routing protocols. It is also the first to address this comparison issue under various radio channel assumptions (e.g. with or without slow or fast fading). Last but not least, it is the first to address the optimal tuning of the MAC parameters within this multihop routing context.

The mathematical framework for the study of the opportunistic routing that is presented in Section VI allows us to formalize our simulation observations. In particular, using this framework we will explain why the tuning of the MAC Aloha parameter that minimizes end-to-end delays over large distances is independent of node density. This is in line with observations made in [6], where it was shown that a lower bound on the mean progress in one hop is independent of the node density in the Poisson model.

\section{Simulation ScEnarios}

\section{A. Time-Space Node Patterns generated by Aloha MAC}

We consider networks formed of nodes randomly distributed on the plane. Specifically, nodes are assumed to be sampled according to some homogeneous Poisson point process with intensity $\lambda$. In practice, the network model considers a finite planar network on the square $[0,1000] \mathrm{m} \times[0,1000] \mathrm{m}$. The locations of the nodes do not change with time slots, but mobility is taken into account in the radio channel model (see model M3 in Section III-B below). In our simulations, the default option is $\lambda=10^{-3}$ nodes $/ \mathrm{m}^{2}$.

Various routing strategies will be combined with the following slotted Aloha scheme: at each time slot, each node tosses a coin with bias $p$, independently of everything else. The nodes tossing heads are the transmitters of this time slot; the other nodes are the receivers.In the following, we call $p$ the transmission probability.

\section{B. Radio Channel}

The power used by all the transmitters is assumed to be equal to some constant $S=1$. We use the following simplified power attenuation function $l(r)=(A r)^{-\beta}$ for some constants $A>0$ and $\beta>2$, which gives the fraction of the emitted power that is received at the distance $r$ from the transmitter. Even if this function has a pole at the origin, it is reasonable and commonly used if the density $\lambda$ of points is not too large or, equivalently, the points are not to close to each other. In the simulations, we take a path-loss exponent $\beta=3$.

In certain models, in addition to the above attenuation function, we assume that the received powers are multiplicatively modified by some location and possibly time dependent random path-loss factors. The following 3 scenarios will be considered.

(M1): Path loss factors are constant equal to 1. This assumption might correspond to a very slow channel fading and/or coding which allows for empirical averaging over fading effects during packet transmission (e.g. based on symbol interleaving).

(M2): Path loss factors are position dependent; they are sampled independently for each transmitter-receiver pair and stay constant for all time slots of the simulation. This corresponds to a slow fading or shadowing effect.

(M3): Path loss factors are position and time dependent; they are sampled independently for each time slot and each transmitter-receiver pair. This might correspond to user mobility and will be the default option in the simulations below.

For models M2 and M3, we assume a Rayleigh fading, where path-loss factors are exponential random variables with parameter 1 (see e.g. [18, p. 50 and 501]). In Section VI we also consider a thermal noise independent of everything else with power denoted by $W$. In the simulations, the default option is $W=0$.

\section{Capture}

Suppose that some station transmits during a given time slot. We assume that it can successfully transmit to some given receiver of this time slot if the SINR ratio at this receiver is not less than some fixed threshold $T$. By the SINR we mean the ratio between the power received from the given transmitter (attenuated and modified by the path-loss factor) and the sum of powers received from all other transmitters of the given time slot, including the power $W$ of the thermal noise. In the simulations, we set $T=10$. 


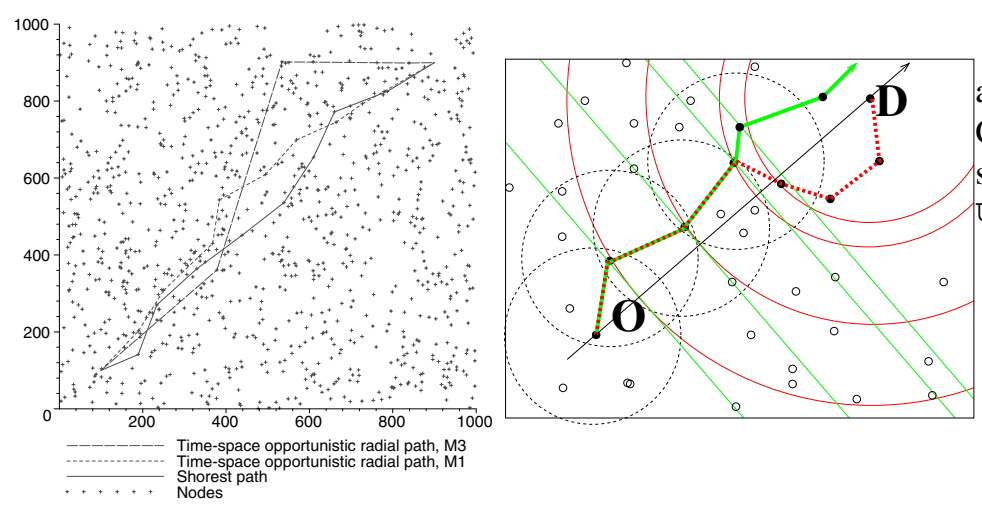

Fig. 3. Left: Samples of routing paths with opportunistic radial routing (with and without fading) and with a shortest path algorithm. Right: When radial and directional schemes diverge.

\section{O-D Pairs and Background Traffic}

In the simulations O-D pairs are selected on opposite parts of the network as shown in Figure 3 (left) with a distance of about $1130 \mathrm{~m}$ from each other. This represents a moderate distance (appr. 9 hops away for a transmission range of $140 \mathrm{~m}$.).

For a fixed O-D pair, and for a given set of network nodes sampled according to a Poisson point process, a basic simulation experiment is the end-to end transmission of one packet of the tagged O-D pair flow, with a tracking of the route selected for this packet, the transmission attempts at each relay node, the end-to-end delay. For the sake of simplicity, in the simulations:

- the tagged packets of the O-D pair are treated as higher priority packets at each node. (We should of course add a queueing delay to account for the competition with cross traffic, but under natural homogeneous traffic assumptions, this should amount to adding a delay with the same law at each node, and should hence not change the main conclusions of the comparison study.);

- all nodes are assumed to always have packets to transmit, and they always transmit whenever authorized by the MAC; these transmissions allow us to take the background traffic into account through the interference they create at each time slot, and in turn, determine which nodes capture the tagged packet transmission.

We repeat a large number of such basic experiments to evaluate means. We consider both packets sent from $\mathrm{O}$ to $\mathrm{D}$ for the same and for different network samples.

\section{E. Routing}

This section presents three routing strategies.

1) Shortest Path Routing: By this we understand routing along the routes with the least number of hops as found by Dijkstra's algorithm [11]. For each given network, this amounts to finding paths of minimal weight between $\mathrm{O}$ and $\mathrm{D}$ in a graph with edges between all pairs of nodes and where the weight of the edge between nodes $x$ and $y$ is 1 if $|x-y| \leq R$ and $\infty$ otherwise, where $R$ is the maximum transmission range and is considered as a parameter of this routing protocol. This shortest path is used for routing all packets of this O-D pair. The Aloha MAC is then used to let the tagged packets progress from $\mathrm{O}$ to $\mathrm{D}$ along this path.
2) Time-space Opportunistic Radial Routing: The algorithms should be described together with the MAC. Consider a tagged packet of the O-D pair flow located at some current node $A$.

Until $A$ is the destination do:

1. Until $A$ tosses heads, end-to-end delay++;

2. When $A$ tosses heads do:

2.1. All the nodes which toss heads (resp. tail) at this time slot are transmitters (resp. receivers);

2.2. The set of transmitters together with the fading variables at that time slot determine the interference everywhere at this time slot;

2.3. The set of receivers $\mathcal{S}$ which satisfy the SINR capture condition at this time slot receive the tagged packet successfully;

2.4. Among the nodes of $\mathcal{S} \cup\{A\}$, the nearest to the destination, say $B$, is the next relay;

2.5. The other nodes of $\mathcal{S}$ discard the tagged packet;

2.6. end-to-end delay++;

2.7. if $A \neq B$ then number-of-hops++;

3. $A:=B$.

A more formal description of this routing protocol, as well as a proof of its convergence, is presented in Section VI. Three examples of radial paths obtained by simulation are given in Figure 3 (left). The path that is the closest to the segment joining the origin to the destination node is obtained with a shortest path routing algorithm. The second path moving farther away from this segment corresponds to the time-space opportunistic radial routing strategy under the M1 model. The third path, which allows one to search for relays very far away from the transmitter corresponds to time-space opportunistic radial routing in the presence of fading (here under the M3 assumptions).

3) Time-space Opportunistic Directional Routing: This is some routing from a given origin node to a destination at infinity in a given direction. It consists in a non-terminating do loop of the same nature as above but with 2.4 replaced by:

2.4' Among the nodes of $\mathcal{S} \cup\{A\}$, the one with the largest abscissa in the given direction, say $B$, is the next relay;

Note that directional routing never delivers a packet to the destination because this destination is at infinity. ${ }^{5}$ However, it is a suitable mathematical model for opportunistic radial packet forwarding between O-D pairs that are separated by a great distance. Indeed, when the remaining distance to the destination is large, then the optimal receiver for the radial path and that for the directional path (see steps 2.4 and $2.4^{\prime}$ ) tend to coincide (cf Figure 3 right).

Since radial routing is the only opportunistic one which delivers the packet to its destination, in what follows when commenting on our simulations, if there is no ambiguity, we will use the term opportunistic routing for radial routing.

\footnotetext{
${ }^{5}$ If one wants to consider the directional routing that delivers the packet to a (finite) destination $D$, then the "given direction" in step 2.4' should be modified at each location of the packet in such a way that it always points towards $D$. Such a routing algorithm may however lead to some oscillations when the packet is close to the destination, and seems to offer no advantages with respect to the radial algorithm. Thus we prefer to consider it only as a mathematical model with $D$ at infinity.
} 


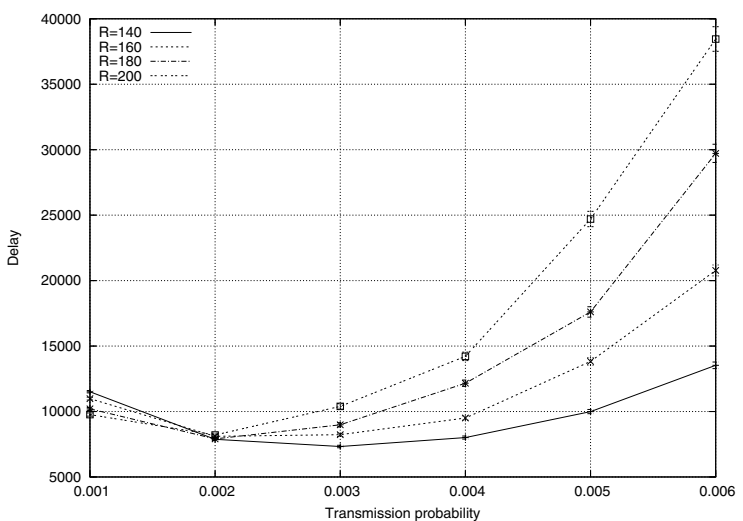

Fig. 4. Shortest path routing algorithm: end-to-end delay versus $p$ for various transmission ranges.

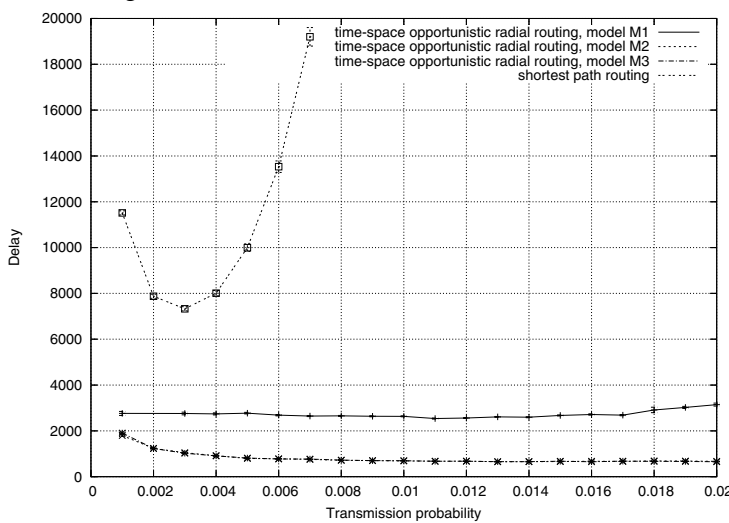

Fig. 5. Comparison between routing strategies: end-to-end delay versus transmission probability $p$.

\section{F. Performance Characteristics}

For a given tagged packet of the O-D pair and a given network of nodes we consider:

- the end-to-end delay, defined as the number of time slots it takes for this packet to go from $\mathrm{O}$ to $\mathrm{D}$,

- the number of hops made by this packet from $\mathrm{O}$ to $\mathrm{D}$,

- the average local delay (delay per hop) defined as the ratio end-to-end-delay/number of hops.

\section{G. Averaging and Confidence Intervals}

In order to calculate the means of the above performance characteristics, we average over 80 different networks connecting a given O-D pair and for each network we average over 5 packets for the O-D pair. The results are always presented with confidence intervals corresponding to a confidence level of $95 \%$. Note that some of these confidence intervals are small and can only be seen when zooming in on the corresponding plots.

\section{Simulation Results}

\section{A. Mean End to End Delay}

For shortest path routing, the maximum transmission range parameter $R$ (recall from Section III-E1 that this is a parameter of Dijkstra's algorithm) has first been optimized in order to make the comparison fair. The end-to-end delays for various values of $R$ and of the transmission probability $p$ are presented in Figure 4. We see that the best delay is obtained with $p=$ 0.003 and with $R=140 \mathrm{~m}$. This value, which is our default value for shortest path routing in what follows, is actually the smallest value of the transmission range which connects the network with high probability in this case.

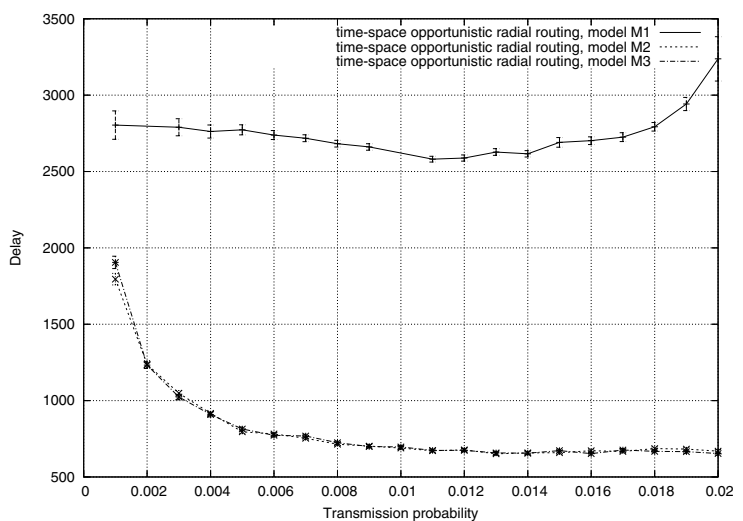

Fig. 6. Effect of fading on time-space opportunistic radial routing: end-to-end delay versus transmission probability $p$.

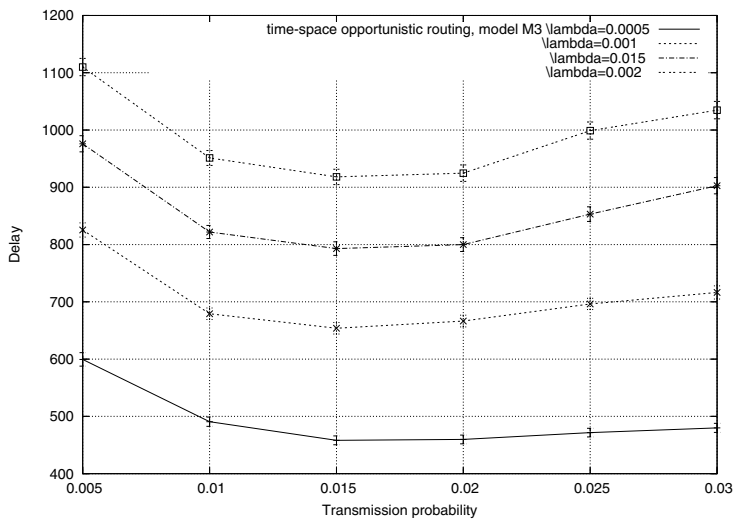

Fig. 7. Time-space opportunistic radial routing; end-to-end delay versus $p$ for various values of the node density.

In Figure 5, we compare the shortest path algorithm and the time-space opportunistic routing. In this figure we give the mean end-to-end delay versus the transmission probability $p$ under different channel fading scenarios. Here is the main observation of the paper.

Observation 4.1: The algorithm based on time-space diversity significantly outperforms the conventional shortest path routing strategy: the average delay of a packet is at least two and a half times smaller for this strategy than for Dijkstra's algorithm.

We also see that the discrepancy between the conventional shortest path routing strategy and time-space opportunistic routing becomes much larger for a large $p$. Moreover, the performance of the opportunistic routing is much less sensitive to a suboptimal choice of the parameter $p$.

Figure 6, which refines Figure 5 for opportunistic routing strategies, shows that:

Observation 4.2: Letting time-space opportunistic routing take advantage of the varying fading (e.g. due to mobility) is beneficial in terms of mean end-to-end delays.

The analysis of the simulation results shows that opportunistic routing in presence of fading (M2 and M3) offer performances roughly four times better in terms of end-to-end delay than opportunistic routing in absence of fading (M1), see Figure 6. The opportunistic routing in slow fading (M2) or in fast fading (M3) offer similar performances. Only very long simulations not presented here allow one to show that opportunistic routing in M3 shows slightly better delays than in M2.

Here is the second most important observation of this paper. Figure 7, which plots the mean end-to-end delay for the M3 time-space opportunistic routing, shows that:

Observation 4.3: There is an optimal value of $p$ that mini- 


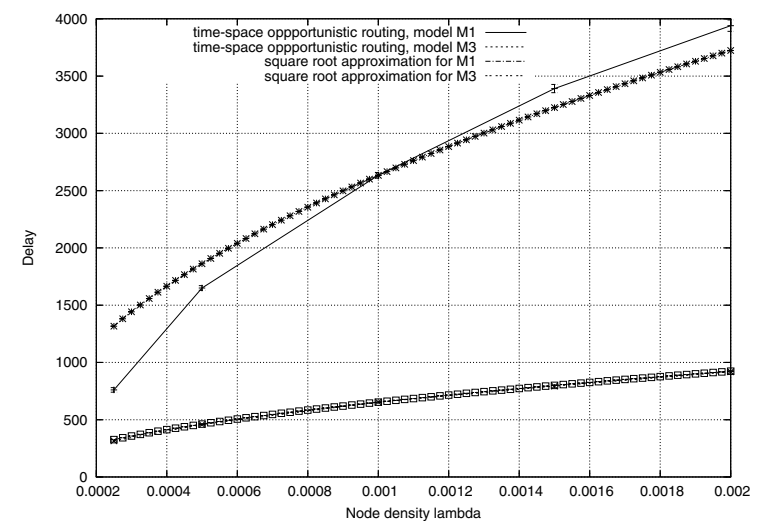

Fig. 8. End to end delay of time-space opportunistic radial routing algorithms (with and without fading) versus node density $\lambda$, comparison with $\sqrt{\lambda}$.

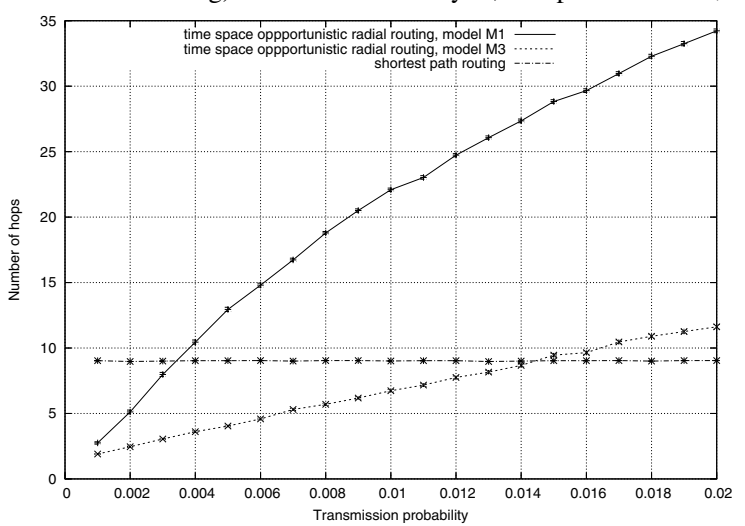

Fig. 9. Mean number of hops from origin to destination with time-space opportunistic radial routing (with and without fading) and with a shortest path routing algorithm.

mizes the mean end-to-end delay of the time-space opportunistic routing algorithm, and that this optimal value $p^{*}$ seems to be the same for all values of the node density $\lambda$.

Similar observations (not presented here) hold for the M1-M2 models described in Section III-B.

In Figure 8 we see that :

Observation 4.4: The mean end-to-end delay of the timespace opportunistic routing algorithm is of the order of $\sqrt{\lambda}$ where $\lambda$ is the node density.

The matching is excellent for opportunistic routing in M3 (and in M2 although it is not shown in Figure 8), for opportunistic routing in M1 there is rough matching. The discrepancy seen for small $\lambda$ may be caused by side effects.

\section{B. Mean Number of Hops, Mean Local Delays}

Figure 9 gives the average number of hops to reach the destination for the two routing strategies with $p$ varying from 0.001 to 0.02 .

Observation 4.5: In the case without fading M1, for small values of $p$, the time-space opportunistic path is shorter (has a smaller mean number of hops) than the Dijkstra shortest path, whereas it is longer for large values of $p$. In the presence of fading, time-space opportunistic routing offers shorter paths than Dijkstra type routing for $p \leq 0.014$ and slightly larger paths than Dijkstra type routing for $p>0.014$.

We also observe that for time-space opportunistic routing, the mean number of hops to reach the destination increases with $p$. This can be easily understood since when $p$ increases, the time-space diversity decreases and thus the number of hops to reach the destination tends to increase.

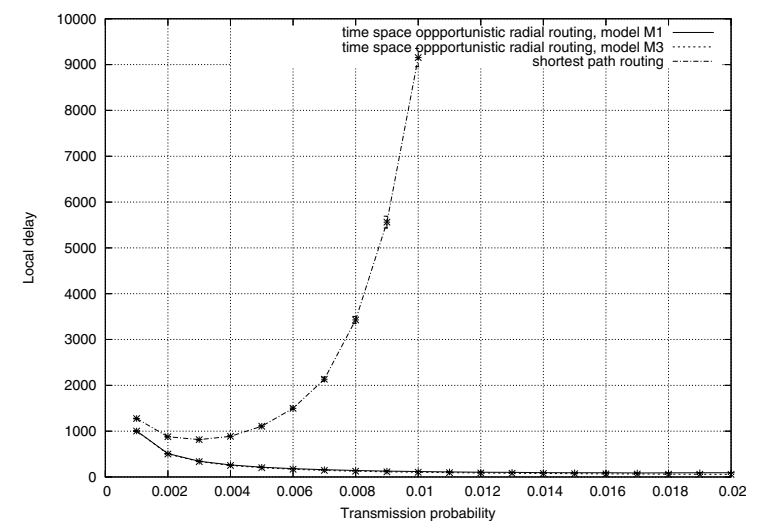

Fig. 10. Mean local delay for time-space opportunistic radial routing (with and without fading) and with a shortest path routing algorithm.

Figure 10 studies the mean local delay for the same three scenarios as above.

Observation 4.6: In time-space opportunistic routing, for each $p$, the mean delay per hop is much smaller than the delay per hop for Dijkstra's algorithm.

This explains why the average delay is smaller for time-space opportunistic routing than for Dijkstra's algorithm even if the number of hops may be larger.

\section{Minimizing End-TO-End Delay for Long Paths}

The aim of this section is to explain Observation 4.3, namely that (and in what sense) there is an optimal value $p^{*}$ that minimizes the mean end-to-end delay of the opportunistic routing for all values of the node density $\lambda$. In our explanation we will use the directional routing model introduced in Section III-E3.

A path can be seen as a sequence of progress segments $\xi_{k}$ for the tagged packet, where the $k$-th segment is that connecting the location of the packet at the $k$-th time slot to its location at the $k+1$-st slot. The $k$-th segment is degenerate and reduces to a point if the node harboring this packet at slot $k$ does not transmit at this time slot or transmits without capture by a node closer to the destination.

Let us define the progress $P_{k}$ of a given progress segment $\xi_{k}$ as the length of the projections of $\xi_{k}$ on the O-D pair direction. Note that for a given O-D pair and a given network of nodes O-D_distance $=\sum_{k=1}^{\text {end-to-end_delay }} P_{k}$. Dividing both sides of this equality by the end-to-end_delay, we obtain

$$
\frac{\text { O-D_distance }}{\text { end-to-end_delay }}=\frac{1}{\text { end-to-end_delay }} \sum_{k=1}^{\text {end-to-end_delay }} P_{k} \text {. }
$$

Suppose now that

$$
\frac{1}{\text { end-to-end_delay }} \sum_{k=1}^{\text {end-to-end_delay }} P_{k} \stackrel{\text { for long radial paths }}{\longrightarrow} \mathcal{P},
$$

where $\mathcal{P}$ is the same constant for all Poisson configurations of nodes and all paths. Then, for long paths and all Poisson configurations of nodes $\frac{\mathrm{O} \text {-D_distance }}{\text { end-to-end_delay }} \approx \mathcal{P}$, and finding the probability $p^{*}$ that minimizes the end-to-end delay for such paths is equivalent to maximizing the constant $\mathcal{P}$.

Conjecture (5.1) is clearly related to the ergodicity of the sequence $\left\{\xi_{k}\right\}$ of segments. Note however that for a fixed O-D pair, the radial routing algorithm delivers the packet to $D$ in a finite number of time slots so that the sequence of progress segments $\xi_{k}$ becomes degenerate after this time. Even before this time, when the remaining distance to the destination is 
small, the progress segments statistically differ from those close to the origin. Consequently, for radial routing, there is no hope of having any stationarity or ergodicity for the sequence of progress segments.

In contrast, directional paths can be expected to have progress segments which become stationary and ergodic. Thus, Observation 4.3 could be explained by the following three properties.

1) Radial and directional routing coincide far from the destination: (cf Figure 3 right).

2) Directional routing produces progress segments which converge to a stationary and ergodic sequence: their Cesaro averages over long paths converge to the mean values of the progress in one slot $\mathcal{P}$ under the steady state regime of the path.

3) The maximization of the constant $\mathcal{P}$ in $p$ is invariant with respect to the intensity of the underlying Poisson point process.

In the next section we will present a mathematical framework, in which these properties can be studied. In particular, we will formalize property 1) and prove property 3). The ergodicity property 2) remains a conjecture at this stage.

\section{Mathematical AnAlysis}

In this section we will study time-space radial and directional routing using the formalism of the theory of point processes.

\section{A. The Model}

Let $\Phi=\left\{X_{i}\right\}_{i}$ be a homogeneous Poisson point process, with points $X_{i} \in \mathbb{R}^{2}$ representing the locations of nodes on the Euclidean plane. We denote by $\lambda$ the intensity of $\Phi$. We will consider two independent sequences $\left\{\mathbf{e}_{i}\right\}_{i},\left\{\mathbf{F}_{i}\right\}_{i}$ of independent marks of the points of $\Phi$.

For all $i$, let $\mathbf{e}_{i}=\left\{e_{i}^{n}\right\}_{n}$ be a sequence of independent and identically distributed (i.i.d.) Bernoulli random variables, where $e_{i}^{n}$ represents the transmission indicator of node $i$ at time $n$, or equivalently the fact that this node tosses heads at time $n$. We assume that $\mathbf{P}\{e=1\}=1-\mathbf{P}\{e=0\}=p$, where $e$ is the generic transmission indicator.

For all $i$, let $\mathbf{F}_{i}=\left\{F_{i, j}^{n}\right\}_{j, n}$ be a family of random vectors, where $F_{i, j}^{n}$ represents the fading between nodes $i$ and $j$ at time $n$. We assume that for each $n$, the variables $\left\{F_{i, j}^{n}\right\}_{i, j}$ are i.i.d. with the same law as a generic variable denoted by $F$ having, if not otherwise specified, a general non-negative distribution with mean 1 . For different $n$ the variables $F_{i, j}^{n}$ need not be independent. We will consider the following 3 scenarios:

(M1) $F_{i, j}^{n} \equiv 1$ for all $i, j, n$,

(M2) $F_{i, j}^{n}=F_{i, j}^{0}$ for all $i, j, n$,

(M3) $F_{i, j}^{n}$ are i.i.d. for all $i, j, n$.

Let $W>0$ be a given random variable representing the thermal noise which is assumed to be independent of $\Phi$. Denote by $l(r)=(A r)^{-\beta}$ the attenuation at distance $r$. Let $S$ denote the (fixed) power used by transmitters.

Let $\Phi_{1}^{n}=\left\{X_{i}: e_{i}^{n}=1\right\}$ denote the point process of transmitters at slot $n$ and $\Phi_{0}^{n}=\left\{X_{i}: e_{i}^{n}=0\right\}$ that of receivers. Suppose that a transmitter is located at $X_{i} \in \Phi_{1}^{n}$.
Consider a receiver located at $X_{j} \in \Phi_{0}^{n}$. Transmitter $X_{i}$ can establish a successful channel to receiver $X_{j}$ if and only if

$$
\frac{S F_{i, j}^{n} l\left(\left|X_{i}-X_{j}\right|\right)}{W+I_{\Phi_{1}^{n} \backslash\left\{X_{i}\right\}}\left(X_{j}\right)} \geq T
$$

where $I_{\Phi_{1}^{n} \backslash\left\{X_{i}\right\}}$ is the shot-noise process of $\Phi_{1}^{n} \backslash\left\{X_{i}\right\}$ :

$$
I_{\Phi_{1}^{n} \backslash\left\{X_{i}\right\}}\left(X_{j}\right)=\sum_{X_{k} \in \Phi_{1}^{n} \backslash\left\{X_{i}\right\}} S F_{k, j}^{n} l\left(\left|X_{k}-X_{j}\right|\right) .
$$

Let $\delta\left(X_{i}, X_{j}, n\right)$ be the indicator that the event (6.1) holds.

When restricted to a bounded window, with $W \equiv 0$ and exponential $F$ in $\mathrm{M} 2$ and M3, the above model corresponds to the simulation scenarios described in Section III.

\section{B. Opportunistic Neighbourhood}

Define the set of neighbors of $X_{i} \in \Phi$ at time $n$ as $\left\{X_{i}\right\}$ plus the set of receivers which capture the packet sent by $X_{i}$ at time $n$ provided $X_{i}$ transmits:

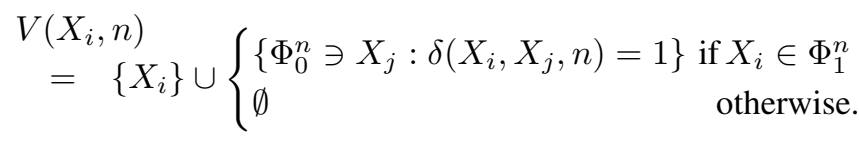

Remark: The above SINR-based notion of neighborhood is quite different from this based on the maximum transmission range (used for the shortest path routing in Section IV). Besides the fact that the neighborhood of a given node is different in different time slots (even in M1 scenario), using (6.1) one can prove (cf [19]) that no receiver at a given time slot can be a neighbour of more than $(1+T) / T$ transmitters. In particular, if $T>1$ then different emitters, no matter how close to each other they are, have disjoint sets of neighbors.

We will now show that the opportunistic neighbourhood is always finite (it is important, both form practical and theoretical point of view) and then calculate the mean number of nodes in some particular case. Note that the assumptions of the following result are satisfied for all considered scenario M1-M3.

Proposition 6.1: Assume that $\mathbf{E}\left[F^{2 / \beta}\right]<\infty$ and that either $\mathbf{E}\left[F^{-2 / \beta}\right]<\infty$ or $\mathbf{E}\left[W^{-2 / \beta}\right]<\infty$. Than for any of the models $M 1-M 3 \mathbf{P}\left\{\# V\left(X_{i}, n\right)=\infty\right.$ for some $\left.i, n\right\}=0$.

Proof: By the Campbell formula [20, page 119], it is enough to prove that $\mathbf{E}^{0}[\# V(0, n)]<\infty$ for fixed $n$, where the expectation $\mathbf{E}^{0}$ is taken with respect to the Palm probability $\mathbf{P}^{0}$. In the case of our independently marked Poisson point process it corresponds to the addition of a node at the origin $X_{0}=0$ endowed with an independent MAC sequence $\mathbf{e}_{0}$ and fading sequence $\mathbf{F}_{0}$ (see Slivnyak's theorem, [20, page 41]). In what follows we consider time $n=0$ and omit it in the notation. Using the Campbell theorem for the second time and the fact that $\Phi_{1}$ and $\Phi_{0}$ are independent Poisson point processes with respective intensities $\lambda p$ and $\lambda(1-p)$, we have

$$
\begin{aligned}
\mathbf{E}^{0} & {[\# V(0)] } \\
& =1+\mathbf{E}^{0}\left[\mathbb{I}\left(e_{0}=1\right) \sum_{i \neq 0} \mathbb{I}\left(X_{i} \in V(0)\right)\right] \\
& =1+p \lambda(1-p) \int_{\mathbb{R}^{2}} \mathbf{E}^{0, x}\left[\delta(0, x) \mid e_{0}=1, e_{x}=0\right] \mathrm{d} x,
\end{aligned}
$$

where the expectation $\mathbf{E}^{0, x}$ is taken with respect to the $t w o$ fold Palm probability $\mathbf{P}^{0, x}$, which in our case corresponds 
to the addition of two nodes, at $X_{0}=0$ and at $X=x$, endowed with independent MAC and fading sequences $\mathbf{e}_{0}, \mathbf{e}_{x}$, $\mathbf{F}_{0}, \mathbf{F}_{x}$. Noting that under $\mathbf{P}^{0, x}$ given $e_{0}=1, e_{x}=0$ the variable $I_{\Phi_{1} \backslash\{0\}}(x)$ has the same distribution as $I_{\Phi_{1}}(0)$ under the stationary distribution $\mathbf{P}$, and passing to polar coordinates, we get from Fubini's theorem that

$$
\begin{aligned}
\int_{\mathbb{R}^{2}} & \mathbf{E}^{0, x}\left[\delta(0, x) \mid e_{0}=1, e_{x}=0\right] \mathrm{d} x \\
= & \mathbf{E}\left[\int_{\mathbb{R}^{2}} \mathbb{I}\left(A|x| \leq T \frac{W+I_{\Phi_{1}}(x)}{S F_{0, x}}\right)^{-1 / \beta} \mathrm{d} x\right] \\
= & \frac{2 \pi}{A} \mathbf{E}\left[\int_{0}^{\infty} r \mathbb{I}\left(r \leq T \frac{W+I_{\Phi_{1}}(0)}{S F}\right)^{-1 / \beta} \mathrm{d} r\right],
\end{aligned}
$$

where $F$ is independent of $W$ and $I_{\Phi_{1}}(0)$. Consequently, we obtain

$\mathbf{E}^{0}[\# V(0)]=1+\frac{p(1-p) \lambda \pi}{A T^{2 / \beta}} \mathbf{E}\left[(S F)^{2 / \beta}\right] \mathbf{E}\left[\left(W+I_{\Phi_{1}}(0)\right)^{-2 / \beta}\right]$

Since we assume $\mathbf{E}\left[F^{2 / \beta}\right]<\infty$ thus the right hand side in the above displayed formula is finite provided $\mathbf{E}\left[W^{-2 / \beta}\right]<\infty$. If this latter condition does not hold, in particular if $W=0$ with some positive probability, we need to prove finiteness of the same negative moment of the shot-noise. For this one can proceed as follows:

$$
\mathbf{E}\left[\left(I_{\Phi_{1}}(0)\right)^{-2 / \beta}\right] \leq \mathbf{E}\left[(S F)^{-2 / \beta}\right] \mathbf{E}\left[\left(\max _{X_{i} \in \Phi_{1}} l\left(\left|X_{i}\right|\right)\right)^{-2 / \beta}\right] .
$$

We assume $\mathbf{E}\left[F^{-2 / \beta}\right]<\infty$ and for our particular attenuation function,

$$
\begin{aligned}
\mathbf{E}\left[\left(\max _{X_{i} \in \Phi_{1}} l\left(\left|X_{i}\right|\right)\right)^{-2 / \beta}\right] & =\int_{0}^{\infty} \mathbf{P}\left\{\max _{X_{i} \in \Phi_{1}} l\left(\left|X_{i}\right|\right) \leq r^{-\beta / 2}\right\} \mathrm{d} r \\
& =\int_{0}^{\infty} \mathbf{P}\left\{\min _{X_{i} \in \Phi_{1}}\left|X_{i}\right| \geq \sqrt{r} / A\right\} \mathrm{d} r \\
& =\int_{0}^{\infty} e^{-\pi \lambda p r / A^{2}} d r=\frac{A^{2}}{\pi \lambda p}<\infty
\end{aligned}
$$

which completes the proof.

Remark: For exponential $F$ in models M2-M3, the expected number of neighbors $\mathbf{E}^{0}[\# V(0)]$ can be calculated explicitly. Indeed, the expectation $\mathbf{E}^{0, x}[\ldots]$ in (6.2) is equal to

$$
\begin{aligned}
\mathbf{E}^{0, x} & {\left[\delta(0, x) \mid e_{0}=1, e_{x}=0\right] } \\
& =\mathbf{E}\left[\exp \left(-\frac{T W}{S l(|x|)}\right)\right] \mathbf{E}\left[\exp \left(-\frac{T I_{\Phi_{1}}(x)}{S l(|x|)}\right)\right] \\
& =\psi_{W}(T / S l(|x|)) \psi_{I_{\Phi_{1}}}(T / S l(|x|)),
\end{aligned}
$$

where $\psi_{W}, \psi_{I_{\Phi}}(\cdot)$ are, respectively, the Laplace transforms of $W$ and $I_{\Phi}=I_{\Phi}(0)$. This last function is known in closed from. In particular, for $W \equiv 0$ we obtain the following formula:

$$
\mathbf{E}^{0}[\# V(0)]=1+\frac{(1-p) \beta}{4 \pi T^{2 / \beta} \Gamma(2 / \beta) \Gamma(1-2 / \beta)},
$$

where $\Gamma(z)=\int_{0}^{\infty} t^{z-1} e^{-t} \mathrm{~d} t$ is the Gamma function, as is easily shown by calculations similar to those in [6], Section III.

\section{Opportunistic Routing}

A point map is a mapping which, for a given realization of the point process $\Phi$, maps each of its points $X_{i} \in \Phi$ to some (possibly the same) point of $\Phi$.
1) Next Relay in Radial Routing: Define the following family of point maps: for $n \geq 0$

$$
\mathcal{A}^{n}\left(X_{i}\right)=\mathcal{A}^{n}\left(X_{i}, \Phi\right)=\arg \min \left\{\left|X_{j}\right|: X_{j} \in V\left(X_{i}, n\right)\right\} .
$$

The above point maps are almost surely well defined due to the well known fact that the probability of finding two or more points of the homogeneous Poisson point process equidistant to the origin is equal to 0 . They represent the motion of a packet from $X_{i}$ at time $n$ to $\mathcal{A}^{n}\left(X_{i}\right)$ in the time-space opportunistic radial routing towards the final destination at the origin 0 of the plane.

In order to describe the route a packet makes form a given point $X \in \mathbb{R}^{2}$ of the plane to the origin 0 lets add these points to the stationary configuration of nodes and denote $\Phi^{0, X}=\Phi \cup\{0, X\}$. Recall that $\Phi^{0, X}$ represents the distribution of nodes under two-fold Palm distribution $\mathbf{P}^{0, X}$. Denote by $\mathbf{e}_{0}, \mathbf{e}_{X}, \mathbf{F}_{0}, \mathbf{F}_{X}$ the MAC and fading marks of nodes at 0 and $X$ under $\Phi^{0, X}$. In the case of independently marked

Poisson point process they are independent of everything else and have the respective generic distributions.

The radial (time-space opportunistic) path of a packet from the source node $X$ at time 0 towards the destination node at the origin 0 of the plane is the sequence of visited nodes $\left\{Y_{n}\right\}_{n}$ defined by:

$$
Y_{0}=X, \quad Y_{n+1}=\mathcal{A}^{n}\left(Y_{n}, \Phi^{X, 0}\right) \text { for } n \geq 0 .
$$

2) Convergence of the Radial Routing: We will say that the time-space opportunistic radial routing algorithm converges if its path is such that $Y_{n} \equiv 0$ after some finite $n$. The following result says that in the presence of the external noise, the (varying) fading is beneficial for the convergence.

Proposition 6.2: Assume that either (i) $W \equiv 0$ or (ii) M3 holds with $F$ having unbounded support (i.e., $\bar{B}(s)=\operatorname{Pr}\{F>$ $s\}>0$ for all $s)$. Then the time-space opportunistic radial routing algorithm converges almost surely under $\mathbf{P}^{0, X}$.

Proof: The convention that $X_{i} \in V\left(X_{i}, n\right)$ implies that no node of norm larger than $\left|Y_{n}\right|$ will ever be selected as the next relay. Hence, for all $n,\left|Y_{n+1}\right| \leq\left|Y_{n}\right|$. In order to prove convergence, it is hence enough to show that the probability that $Y_{n+k}=Y_{n}$ for all $k \geq 1$ and for some $n$ is 0 when $Y_{n} \neq$ 0 . Assume M3 holds. Denote by $\mathcal{G}$ the $\sigma$-algebra generated by $\Phi$. Conditionally on $\mathcal{G}$ and on the event $Y_{n}=X_{i} \neq 0$ for a given $X_{i} \in \Phi \cup\{X\}$, we have

$$
\begin{aligned}
\mathbf{P}^{0, X}\left\{Y_{n}=Y_{n+1}=\ldots=Y_{n+k} \mid \mathcal{G}, Y_{n}=X_{i} \neq 0\right\} \\
=\prod_{i=0}^{k-1} \mathbf{P}^{0, X}\left\{Y_{n+i}=Y_{n+i+1} \mid \mathcal{G}, Y_{n+i}=X_{i} \neq 0\right\} \\
=\left(\mathbf{P}^{0, X}\left\{Y_{n}=Y_{n+1} \mid \mathcal{G}, Y_{n}=X_{i} \neq 0\right\}\right)^{k}
\end{aligned}
$$

so that it is enough to prove that

$$
\mathbf{P}^{0, X}\left\{Y_{n+1}=Y_{n} \mid \mathcal{G}, Y_{n}=X_{i} \neq 0\right\}<1
$$

to conclude the proof. But we have

$$
\begin{aligned}
\mathbf{P}^{0, X}\left\{\left|Y_{n+1}\right|<\left|Y_{n}\right| \quad \mathcal{G}, Y_{n}=X_{i} \neq 0\right\} & \\
\geq & \mathbf{P}^{0, X}\left\{\mathcal{A}^{n}\left(X_{i}, \Phi\right)=0 \quad \mathcal{G}\right\} \\
= & p(1-p) \mathbf{P}^{0, X}\left\{\frac{S F_{i, 0}^{n} l\left(\left|X_{i}\right|\right)}{W+I_{\Phi_{1}^{n} \backslash\left\{X_{i}\right\}}(0)} \geq T\right. \\
& \left.\mid \mathcal{G}, e_{i}^{n}=1, e_{0}^{n}=0\right\} .
\end{aligned}
$$


Since $F_{i, 0}^{n}$ is independent of $I_{\Phi_{1}^{n} \backslash\left\{X_{i}\right\}}(0)$ and $W$, the probability $\mathbf{P}^{0, X}\{\ldots\}$ in the last formula can be expressed as

$$
\mathbf{E}^{0, X}\left[\bar{B}\left(\frac{T\left(W+I_{\Phi_{1}^{n} \backslash\left\{X_{i}\right\}}(0)\right)}{S l\left(\left|X_{i}\right|\right)}\right) \mid \mathcal{G}, e_{i}^{n}=1, e_{0}^{n}=0\right]
$$

and is positive by the assumption $\bar{B}(s)>0$ and the fact that $I_{\Phi_{1}^{n} \backslash\left\{X_{i}\right\}}(0)<\infty$ a.s. as a Poisson shot-noise. This concludes the proof of case (ii).

Consider now the case (i) $W \equiv 0$. Let $\mathcal{H}$ denote the $\sigma$ algebra generated by $\Phi$ and the fading variables (under M1 or M2, these variables do not vary over time). Using the same argument as before it is enough to prove that

$$
\mathbf{P}^{0, X}\left\{Y_{n+1}=Y_{n} \mid \mathcal{H}, Y_{n}=X_{i} \neq 0\right\}<1 .
$$

And we have

$$
\begin{aligned}
\mathbf{P}^{0, X}\left\{\left|Y_{n+1}\right|<\left|Y_{n}\right| \quad \mathcal{H}, Y_{n}=X_{i} \neq 0\right\} \\
\geq \mathbf{P}^{0, X}\left\{\mathcal{A}^{n}\left(X_{i}, \Phi\right)=0 \quad \mid \mathcal{H}\right\} \\
=\mathbf{P}^{0, X}\left\{\frac{S F_{i, 0} l\left(\left|X_{i}\right|\right)}{I_{\Phi_{1}^{n} \backslash\left\{X_{i}\right\}}(0)} \geq T, e_{i}^{n}=1, e_{0}^{n}=0 \mid \mathcal{H}\right\} \\
=p(1-p) \mathbf{P}^{0, X}\left\{I_{\Phi_{1}^{n} \backslash\left\{X_{i}\right\}}(0) \leq \frac{S F_{i, 0} l\left(\left|X_{i}\right|\right)}{T}\right. \\
\left.\qquad \mid \mathcal{H}, e_{i}^{n}=1, e_{0}^{n}=0\right\} .
\end{aligned}
$$

The proof then follows from the fact that the $\mathcal{H}$-conditional law of the Poisson shot-noise process $I_{\Phi_{1}^{n} \backslash\left\{X_{i}\right\}}(0)$ puts a positive mass on the interval $[0, z]$ for all positive $z$.

Remark: Note that result of Proposition 6.2 cannot be immediately concluded from the fact that at any time and current location of the packet there is a positive probability of delivering it directly to the destination. In fact, our routing protocol is not allowed to wait for such an event. We remark also, that under M1 and M2 with $W>0$, the is a non-negative probability that the packet is trapped forever at some isolated node.

3) Directional Path: In order to study the routing on long OD distances it is customary to introduce another point map. Denote by $\langle x, y\rangle$ the scalar product in $\mathbb{R}^{2}$ and for a given unit vector (think of a "direction") $\mathrm{d} \in \mathbb{R}^{2},|\mathrm{~d}|=1$, define

$$
\mathcal{A}_{\mathrm{d}}^{n}\left(X_{i}\right)=\arg \max \left\{\left\langle X_{j}, \mathrm{~d}\right\rangle: X_{j} \in V\left(X_{i}, n\right)\right\} .
$$

It is well known that the probability of finding two or more points of a homogeneous Poisson point process on a line with a given direction is equal to 0 . Moreover, under assumptions of Proposition 6.1 the point maps $\mathcal{A}_{\mathrm{d}}^{n}$ are well defined.

Consider $\Phi^{X}=\Phi \cup\{X\}$ and let the node at $X$ be marked by an independent MAC sequence $\mathbf{e}_{X}$ and fading sequence $\mathbf{F}_{X}$. The d-directional path followed by a packet routed from $X$ in the direction $\mathrm{d}$ by the time-space opportunistic directional routing algorithm is the sequence $\left\{Z_{n}=Z_{n}(X)\right\}_{n \geq 0}$ defined by

$$
Z_{0}=X, \quad Z_{n+1}=\mathcal{A}_{\mathrm{d}}^{n}\left(Z_{n}, \Phi^{X}\right) \text { for } n \geq 0 .
$$

The reason for which we introduce the directional roting is that the radial paths should converge to these of the directional one when the distance between the origin and the destination grows to infinity. This property can be formalized in the following way: The finite-dimensional distributions of the sequence $\left\{Y_{n}(X)-X\right\}_{n}$ under $\mathbf{P}^{0, X}$ converge weakly to those of $\left\{Z_{n}(0)\right\}$ under $\mathbf{P}^{0}$ when $|X| \rightarrow \infty$ such that $-X /|X|=\mathrm{d}$. Roughly speaking this result is due to the fact that the optimal choices in "arg min" in $\mathcal{A}$ and "arg max" in $\mathcal{A}_{\mathrm{d}}$ coincide with high probability when the packet is far from the destination. A formal proof that could follow the lines of [21, Lemma 1,Theorem 1], is omitted due to space constraints. The directional routing reveals interesting scaling properties.

\section{Scaling Properties of the Directional Paths}

In connection with Observation 4.4, we prove the following result where $\mathbf{P}^{0}=\mathbf{P}_{\lambda}^{0}$ denotes the probability measure under which the underlying Poisson point process $\Phi$ has intensity $\lambda$.

Proposition 6.3: Assume that $W \equiv 0$ and $\mathbf{E}[F 2 / \beta]<\infty$, $\mathbf{E}\left[F^{-2 / \beta}\right]<\infty$ so as directional path $\left\{Z_{n}\right\}$ to be well defined. Then for any of the models $M 1-M 3$, the law of the sequence $\left\{Z_{n}=Z_{n}(0)\right\}_{n}$ under $\mathbf{P}_{\lambda}^{0}$ is the same as that of $\left\{Z_{n} / \sqrt{\lambda}\right\}_{n}$ under $\mathbf{P}_{1}^{0}$.

Proof: Note that the distribution of the underlying Poisson point process $\left.\Phi=\left\{X_{i}\right)\right\}_{i}$ under $\mathbf{P}_{\lambda}^{0}$ is the same as the distribution of $\Phi(\lambda)=\left\{\left(X_{i} / \sqrt{\lambda}\right)\right\}_{i}$ under $\mathbf{P}_{1}^{0}$. Moreover, under our assumptions on $l$ and $W=0$, the SINR (in fact SIR) is invariant with respect to the scaling $\Phi(\lambda)$ of the point process. Indeed, $l\left(\left|X_{i} / \sqrt{\lambda}-X_{j} / \sqrt{\lambda}\right|\right)=\lambda^{\beta / 2} l\left(\left|X_{i}-X_{j}\right|\right)$ and $I_{\Phi_{1}^{n}(\lambda) \backslash\left\{X_{i} / \sqrt{\lambda}\right\}}\left(X_{j} / \sqrt{\lambda}\right)=\lambda^{\beta / 2} I_{\Phi_{1}^{n} \backslash\left\{X_{i}\right\}}\left(X_{j}\right)$. Moreover, the dilation (our scaling) is a conformal mapping (preserves angles). Consequently, the directional point map $\mathcal{A}_{\mathrm{d}}^{n}\left(X_{i} / \sqrt{\lambda}\right)$ acting on $\Phi^{0}(\lambda)$ is equal to $1 / \sqrt{\lambda} \mathcal{A}_{\mathrm{d}}^{n}\left(X_{i}\right)$ acting on $\Phi_{1}^{0}$. This completes the proof.

For fixed $n$, consider now the optimization of the mean progress of the directional path in $n$ hops with respect to the transmission probability $p$ :

$$
p^{*}(n, \lambda)=\underset{0 \leq p \leq 1}{\arg \max } \mathbf{E}_{\lambda}^{0}\left[\left\langle Z_{n}, \mathrm{~d}\right\rangle\right] .
$$

The following corollary is a simple consequence of Proposition 6.3 (cf Observation 4.3).

Corollary 6.4: Under the assumptions of Proposition 6.3 the optimal transmission probability $p^{*}(n, \lambda)=p^{*}(n)$ does not depend on $\lambda$.

Similarly, conjecturing the ergodicity property of the increments of $\left\{Z_{n}\right\}$ under the assumptions of Proposition 6.3 the transmission probability $p^{*}$ that optimizes the ergodic mean progress $\mathcal{P}$ of the directional routing in one hop does not depend on $\lambda$.

\section{E. Other Point Maps and their Optimal Transmission Proba- bilities}

Several other point maps can be used or were already used for routing. Rephrased with the terminology of the present paper, the authors of [6] used the following directional point map:

$$
\tilde{\mathcal{A}}_{\mathrm{d}}\left(X_{i}\right)=\underset{X_{j} \in \Phi_{0}}{\arg \max }\left\{\left\langle X_{j}-X_{i}, \mathrm{~d}\right\rangle p_{\left|X_{j}-X_{i}\right|}\right\},
$$

where $p_{|x|}=\mathbf{E}^{0, x}\left[\delta(0, x) \mid e_{x}=0, e_{0}=1\right]$ is the probability of successful transmission from 0 to $x$. Note that this point map is less adaptive (more parametric) than $\mathcal{A}_{\mathrm{d}}$ as it does not take advantage of the actual state of the SINR conditions at the receivers but only of their distance to the emitter; 
the indicator of successful reception is replaced there by the reception probability at a given location.

The distribution function of the associated progress in one hop: $\tilde{P}_{1}=\max _{X_{j} \in \Phi_{0}}\left\{\left\langle X_{j}, \mathrm{~d}\right\rangle p_{\left|X_{j}\right|}\right\}$ was calculated under $\mathbf{P}^{0}$ under conditions which can be rephrased as:

(M4) $F_{i, j}^{n}=F_{i}^{n}$ for all $i, j, n$ and $F_{i}^{n}$ are i.i.d. exponential with mean 1 .

Under these conditions it was shown that the mean progress $\mathbf{E}^{0}\left[P_{1}\right]$ offered in one time slot by the directional routing $\mathcal{A}_{\mathrm{d}}$ is not smaller than $\mathbf{E}^{0}\left[\tilde{P}_{1}\right]$ offered by $\tilde{\mathcal{A}}_{\mathrm{d}}$. Moreover, $\mathbf{E}^{0}\left[\tilde{P}_{1}\right]$ was shown to scale like $1 / \sqrt{\lambda}$ and to be maximized by a value of the transmission probability $\tilde{p}^{*} \approx 0.05$. It was argued in [6] that such a one-hop optimization of $\tilde{A}_{\mathrm{d}}$ is sufficient if the locations of nodes are independently re-sampled in each time slot. This last scenario is similar in its spirit to the Poisson Weighted Infinite Tree model of [22], and was argued to be reasonable if nodes are highly mobile; this model is easier to analyze as the successive hops of the routing become i.i.d. (thus ergodicity is granted). In consequence, in this "highly mobile" scenario the optimization of the mean progress $\mathbf{E}^{0}\left[\tilde{P}_{1}\right]$ in one slot minimizes the mean end-to-end delay over a long multi-hop radial path.

For the setting of Section IV, the optimal transmission probability is $p^{*} \approx 0.014$ for $\mathrm{M} 1$ and $p^{*} \approx 0.018$ for M2 and M3. These optimal values slightly differ from the optimal transmission probability $\tilde{p}^{*} \approx 0.05$ obtained in [6] for M4 with the same parameter setting $(\beta=3, W=0, T=10)$. The discrepancy can be explained by the differences alluded to above: a less adaptive point map $\tilde{\mathcal{A}}_{\mathrm{d}}$ and different radio channel assumptions.

\section{CONCLUSION}

We have used simulations to show that time-space opportunistic routing schemes significantly improve the performance of multi-hop networks compared to conventional shortest path routing algorithms. The gain in terms of average delay incurred by a packet traveling from a source to a distant destination node is at least 2.5 depending on the actual network parameters. The performance of the opportunistic routing is much less sensitive to a suboptimal choice of the parameter $p$. Moreover, we argue that an optimal tuning of the MAP $p$ of the Aloha MAC may be independent of node density.

We have also proposed a new mathematical framework to prove some of the observations made by simulation. In particular, this framework allowed us to prove that these routing algorithms can be optimized so as to minimize the average end-to-end delay incurred by a packet over long paths and that the optimum transmission probability does not depend on the node density in the random homogeneous case. The potential of this mathematical framework is well illustrated by the fact that the scaling property of Proposition 6.3 and the invariance property of Corollary 6.4 remain true for more general scenarios, e.g. when the routing is defined by more general classes of point maps.

Various challenging problems remain open. On the practical side, we would quote in particular the evaluation of the overhead associated with such schemes. The simulation study also lead to several conjectures. The ergodicity of the point maps introduced in connection with these routing algorithms is probably the most interesting open mathematical problem of the paper.

\section{REFERENCES}

[1] C. Perkins, E. Belding-Royer, and S. Das, "Ad hoc On-demand Distance Vector (AODV) routing," July 2003, rFC 3561, Experimental.

[2] D. B. Johnson, D. A. Maltz, and Y.-C. Hu, "The Dynamic Source Routing protocol for mobile ad hoc networks (DSR)," July 19 2004, internetDraft, draft-ietf-manet-dsr-10.txt, work in progress.

[3] C. Adjih, T. Clausen, P. Jacquet, A. Laouiti, P. Minet, P. Muhlethaler, A. Qayyum, and L. Viennot, "Optimized link-state routing protocol," RFC 3626, October 2003

[4] R. Ogier, F. Templin, and M. Lewis, "Topology dissemination Based on Reverse-Path Forwarding (TBRPF)," February 2004, rFC 3684, Experimental.

[5] B. Blum, T. He, S. Son, and J. Stankovic, "IGF: A state-free robust communication protocol for wireless sensor networks," University of Virginia CS Department, Tech. Rep. CS-2003-1, 2003.

[6] F. Baccelli, B. Blaszczyszyn, and P. Mühlethaler, "An Aloha Protocol for Multihop Mobile Wireless Networks," in Proceedings of the Allerton Conference. Urbana Champaign: University of Illinois, November 2003, and IEEE Transactions on Information Theory, 52(2):421-436, 2006.

[7] S. Biswas and R. Morris, "Exor: opportunistic multi-hop routing for wireless networks," in Proceedings SIGCOMM '05. New York, NY, USA: ACM, 2005, pp. 133-144.

[8] N. Abramson, "The Aloha system - another alternative for computer communication," in Proc. of AFIPS, 1970, pp. 295-298.

[9] P. Gupta and P. R. Kumar, "The capacity of wireless networks," IEEE Transactions on Information Theory, vol. 46, no. 2, pp. 388-404, 2000.

[10] J. Kurose and K. Ross, Computer Networking. A Top-Down Approach Featuring the Internet. Addison Wesley, 2001.

[11] E. W. Dijkstra, "A note on two problems in connexion with graphs," Numerische Mathematik, vol. 1, pp. 269-271, 1959.

[12] B. Karp, "Geographic routing for wireless networks," Harvard University," $\mathrm{PhD}$ thesis, 2000. [Online]. Available: citeseer.nj.nec.com/karp00geographic.html

[13] G. G. Finn, "Routing and addressing problems in large metropolitanscale internetworks," Information Sciences Institute, Tech. Rep. ISI/RR87-180, March 1987.

[14] I. Stojmenovic and X. Lin, "Loop-free hybrid single-path/flooding routing algorithms with guaranteed delivery for wireless networks," IEEE Transaction on Parallel and Distributed Systems, vol. 12, pp. 1023-1032, 2001.

[15] — , "Power-aware localized routing in wireless networks," IEEE Transaction on Parallel and Distributed Systems, vol. 12, no. 11, pp. 1122-1133, 2001.

[16] B. Karp and H. T. Kung, "Gpsr: Greedy perimeter stateless routing for wireless networks," in MobiCom 2000. Boston MA: ACM/IEEE, August 2000, pp. 243-254.

[17] F. Baccelli, B. Błaszczyszyn, and P. Mühlethaler, "An optimized relay self selection technique in opportunistic routing in mobile ad hoc networks," 2008, submitted.

[18] D. Tse and P. Viswanath, Foundamentals of Wireless Communication. Cambridge University Press, 2005.

[19] F. Baccelli and B. Blaszczyszyn, "On a coverage process ranging from the boolean model to the poisson voronoi tessellation, with applications to wireless communications," Adv. Appl. Prob. SGSA, vol. 33, no. 2, pp. 293-323, 2001.

[20] D. Stoyan, W. Kendall, and J. Mecke, Stochastic Geometry and its Applications. Chichester: Wiley, 1995.

[21] C. Bordenave, "Navigation on a poisson point process," INRIA, Tech. Rep. RR-5790, 2006. [Online]. Available: http://www.citebase.org/abstract?id=oai:arXiv.org:math/0601122

[22] D. Aldous and J. Steel, "The objective method : Probabilistic combinatorial optimization and local weak convergence," 2002, in preparation. [Online]. Available: citeseer.ist.psu.edu/aldous02objective.html 